\title{
High expression of AGBL2 is a novel prognostic factor of adverse outcome in patients with ovarian carcinoma
}

\author{
WEI-PENG HE ${ }^{1}$ and LI-LI WANG ${ }^{2}$ \\ ${ }^{1}$ Department of Gynecology, The First Affiliated Hospital, Sun Yat-Sen University, Guangzhou, \\ Guangdong 510080; ${ }^{2}$ State Key Laboratory of Oncology in South China, Cancer Center, \\ Sun Yat-Sen University, Guangzhou, Guangdong 510060, P.R. China
}

Received March 7, 2019; Accepted August 16, 2019

DOI: $10.3892 / \mathrm{ol} .2019 .10829$

\begin{abstract}
The putative oncogenic role of ATP/GTP binding protein like 2 (AGBL2) in catalyzing $\alpha$-tubulin detyrosination has recently been characterized in cancer. However, the status of AGBL2 expression in ovarian cancer and its potential clinical and prognostic significance remain unclear. In the present study, immunohistochemistry staining investigated the protein expression level of AGBL2 in paraffin-embedded pathological specimens from 30 normal ovaries, 35 ovarian cystadenomas, 38 borderline ovarian tumors and 165 invasive ovarian carcinomas. The association between AGBL2 expression and clinicopathological characteristics of patients was evaluated using the $\chi^{2}$ test or Fisher's exact test. The survival status of patients was assessed by receiver-operator curve analysis. The results demonstrated that high expression of AGBL2 was observed in 9\% of cystadenomas cases, $21 \%$ of borderline tumors cases and 38\% of ovarian carcinomas cases; however AGBL2 expression was not high in normal ovarian tissues $(\mathrm{P}<0.01)$. Furthermore, the results demonstrated that high expression of AGBL2 was associated with tumor histological grade, advanced $\mathrm{pT} / \mathrm{pN} / \mathrm{pM}$ and cancer stage according to the International Federation of Gynecology and Obstetrics $(\mathrm{P}<0.05)$. Following univariate survival analysis of the ovarian carcinoma groups, high expression of AGBL2 was significantly associated with shorter patient survival $(\mathrm{P}<0.001)$. In addition, multivariate analysis revealed that AGBL2 could be identified as a potential independent prognostic factor for overall survival in patients with ovarian carcinoma $(\mathrm{P}=0.004)$.
\end{abstract}

Correspondence to: Dr Wei-Peng He, Department of Gynecology, The First Affiliated Hospital, Sun Yat-Sen University, 58 Zhongshan Road II, Guangzhou, Guangdong 510080, P.R. China

E-mail: weipenghe@126.com

Dr Li-Li Wang, State Key Laboratory of Oncology in South China, Cancer Center, Sun Yat-Sen University, 651 Dongfeng Road East, Guangzhou, Guangdong 510060, P.R. China

E-mail: wanglili1211@hotmail.com

Key words: ovarian carcinoma, ATP/GTP binding protein like 2, immunity related GTPase M, immunohistochemistry, prognosis
Furthermore, the results demonstrated that AGBL2 expression was significantly associated with the expression of immunity related GTPase M (IRGM) ( $\mathrm{P}=0.013)$ and LC3A/B ( $\mathrm{P}=0.004)$. IRGM expression level was also significantly associated with LC3A/B expression level $(\mathrm{P}=0.023)$. These findings demonstrated that AGBL2 expression was high in ovarian carcinomas, which suggested that AGBL2 may participate in the acquisition of an aggressive phenotype and may therefore serve as an independent prognostic molecular marker.

\section{Introduction}

Ovarian carcinoma is one of the most lethal malignancies of the female reproductive system worldwide $(1,2)$. The incidence of ovarian carcinoma has gradually increased in Asian countries, particularly in Japan (from 3.6 to 6.5\%) in recent years (3). Despite recent advances in surgical techniques and medical management (4-6), the long-term prognosis of patients with ovarian carcinoma remains unsatisfactory. Furthermore, due to the lack of early diagnostic methods and characteristic symptoms, most patients with ovarian carcinoma are diagnosed at advanced clinical stages [stages III and IV according to the International Federation of Gynecology and Obstetrics (FIGO)] and have therefore a poor prognosis. The overall 5-year survival rate of patients with ovarian carcinoma is $\sim 20 \%$ (7). It is therefore crucial to determine novel biomarkers suitable for tumor early detection, prediction of tumor behavior and targeted therapy.

AGBL2 is a cytoplasmic carboxypeptidase that catalyzes microtubule (MT) detyrosination (8). MTs are polarized polymers comprising $\alpha / \beta$ tubulin heterodimers (9). Multiple posttranslational modifications on tubulins, including detyrosination, can regulate the stability of MTs (10). MT detyrosination, which targets the C-terminal tyrosine residual of $\alpha$-tubulin and increases MT stability (11), is frequently observed in human malignances, such as neuroblastomas, breast cancer and prostate cancer (12-14). In cancer cells, detyrosinated tubulins assemble to form an invasive front and microtentacles $(15,16)$. Furthermore, detyrosinated tubulins are more resistant to microtubule-targeting agents (17). Previous studies demonstrated that AGBL2 promotes tumorigenesis and cancer progression in breast cancer, prostate cancer and hepatocellular carcinoma $(8,18)$ and could be considered as a potential biomarker for lymph node metastasis 
and chemotherapy resistance in breast cancer (19). In addition, AGBL2 promotes hepatocellular carcinoma cancer cell growth via IRGM-regulated autophagy. After knockdown of IRGM, AGBL2-induced up-regulation of the crucial members in autophagy (i.e. LC3A/B, ATG5, ATG12 and ATG3) was mainly abolished (18).

AGBL2 expression and its clinical and prognostic relevance in ovarian carcinomas have not yet been elucidated. In the present study, expression status of AGBL 2 protein was detected by immunohistochemistry (IHC) in a series of ovarian cancer specimens. Furthermore, the clinicopathological and prognostic significance of AGBL2 protein expression in ovarian carcinoma tissues was evaluated. To the best of our knowledge, the present study was the first to provide novel insights into the role of AGBL2 in the development and progression of ovarian carcinoma.

\section{Materials and methods}

Patients and tissue specimens. Archived paraffin-embedded cancer specimens were obtained from 268 patients who underwent initial surgical resection between June 2002 and June 2010. All samples, including 30 normal ovarian tissues, 35 cystadenoma tissues, 38 borderline ovarian tumors tissues and 165 invasive ovarian carcinoma tissues, were collected from the archives of the Department of Pathology from the Cancer Center and the First Affiliated Hospital (Sun Yat-Sen University). No patient had received preoperative chemoradiation therapy and all patients had completed clinical follow-up (up to December 2016), of which data were available for review in this study. Patients with incomplete clinical follow-up data or who died from unknown causes were excluded from the study. In addition, patient survival time data and clinicopathological characteristics were collected. The age of the 165 patients diagnosed with primary ovarian carcinoma ranged from 19 to 85 years (mean age, 51.0 years) and their clinicopathological characteristics are presented in Table I. Patients provided informed consent and the study was approved by the Institute Research Medical Ethics Committee of Sun Yat-Sen University.

Construction of tissue microarrays (TMA). Tissue microarrays were constructed as previously described $(20,21)$. Paraffin-embedded tissue blocks and the corresponding histological hematoxylin and eosin (H\&E) stained slides were overlaid for TMA sampling construction. Typical lesions (cancer-rich) were identified by a senior pathologist. Corresponding histological H\&E stained slide of each paraffin-embedded tissue was examined under a BX-60 optical microscope (Olympus Corporation) and marked for representative areas of tumor tissues. Subsequently, a tissue microarray instrument (Beecher Instruments, Inc.) was used to obtain tissue cylinders of $\sim 0.6-\mathrm{mm}$ in diameter from selected cancer areas of patient tissue. These sections were then re-embedded into a recipient paraffin block with hollow presets. TMA block was then cut into slices (5 $\mu \mathrm{m}$ thick) and mounted onto microscope slides.

IHC staining. IHC staining was performed using a standard streptavidin-biotin-peroxidase complex method as previously described (22). Briefly, tissue sections were deparaffinized and rehydrated in an ethanol gradient $(100,90,75$ and 50\%). The endogenous peroxidase activity was blocked with $3 \% \mathrm{H}_{2} \mathrm{O}_{2}$ at room temperature for $10 \mathrm{~min}$. Slides were immersed in $10 \mathrm{mM}$ citrate buffer ( $\mathrm{pH}$ 8.0) and boiled for $3 \mathrm{~min}$ in a pressure cooker for antigen retrieval. Non-specific binding was blocked with $5 \%$ normal goat serum (OriGene Technologies, Inc.) for $10 \mathrm{~min}$ at room temperature. Slides were incubated with polyclonal primary antibodies against AGBL2 (1:50; cat. no. HPA007718; Abcam), IRGM (1:200; cat. no. ab69494; Abcam) and LC3A/B (1:100; cat. no. 12741; CST Biological Reagents Co., Ltd.) at $4^{\circ} \mathrm{C}$ overnight in a moist chamber. Slides were then incubated with biotinylated goat anti-mouse immunoglobulin $\mathrm{G}$ (IgG) antibody (1:100; cat. no. sc-516102; Santa Cruz Biotechnology, Inc.) for $30 \mathrm{~min}$ at room temperature followed by a streptavidin-peroxidase conjugate also for $30 \mathrm{~min}$ at room temperature. Isotope-matched human IgG was used in each case as a negative control. The 3,5-diaminobenzidine Substrate kit (Dako; Agilent Technologies, Inc.) was used for color development followed by Mayer hematoxylin counterstaining. Slides were counterstained with $10 \%$ Mayer's hematoxylin for $1 \mathrm{~min}$. AGBL2 staining was evaluated according to previously described protein expression criteria (14).

Statistical analysis. Statistical analysis was carried out using SPSS statistical software version 20.0 (IBM Corp.). The association between AGBL2 expression and clinicopathological features of patients with ovarian carcinoma was assessed using the $\chi^{2}$-test or Fisher's exact test. For univariate survival analysis, survival curves were obtained through the Kaplan-Meier method. Log rank test was used to compare different survival curves. Multivariate survival analysis was carried out on all parameters that were found to be significant following univariate analysis by using Cox regression model. The differences between the clinicopathological characteristics and survival predictions were estimated using receiver operating characteristic curve (ROC) analysis. $\mathrm{P}<0.05$ was considered to indicate a statistically significant difference.

\section{Results}

AGBL2 expression in ovarian tissues. The results from IHC demonstrated that AGBL2 positive staining was mainly detected in the cytoplasm of ovarian epithelial cells; however, AGBL2 was occasionally visible in the nuclear (Fig. 1). High expression of AGBL2 was observed in 62/165 (38\%) of ovarian carcinomas (Fig. 1D), in 8/38 (21\%) of borderline tumors and in $3 / 35$ (9\%) of cystadenomas (Fig. 1C), but not in normal ovarian tissues (Fig. 1B). In addition, the results demonstrated that the increased frequency of highly expressed AGBL2 in ovarian carcinomas was statistically significant $(\mathrm{P}<0.01$; Table II) compared with AGBL2 expression in benign and borderline tumors.

Association of AGBL2 expression with the clinicopathologic characteristics and survival of patients with ovarian carcinoma. Following AGBL2 expression analysis, the clinicopathological significance of highly expressed AGBL2 in patients with ovarian carcinoma was analyzed. The results demonstrated that high AGBL2 expression was positively associated with high histological grade, advanced 
Table I. Association between AGBL2 expression and the clinicopathological characteristic of 165 patients with ovarian carcinoma.

AGBL2 protein

\begin{tabular}{|c|c|c|c|c|}
\hline Variable & Number of cases, $\mathrm{n}$ & Low expression, $\mathrm{n}(\%)$ & High expression, $\mathrm{n}(\%)$ & P-value \\
\hline Age at surgery, years & & & & 0.764 \\
\hline$\leq 51.0^{\mathrm{b}}$ & 88 & $54(61)$ & $34(39)$ & \\
\hline$>51.0^{\mathrm{b}}$ & 77 & $49(64)$ & $28(36)$ & \\
\hline Histological type & & & & 0.659 \\
\hline Serous & 109 & $69(63)$ & $40(37)$ & \\
\hline Mucinous & 19 & $13(68)$ & $6(32)$ & \\
\hline Others $^{c}$ & 37 & $21(57)$ & $16(43)$ & \\
\hline Histological grade (Silveberg) & & & & 0.040 \\
\hline G1 & 28 & $22(79)$ & $6(21)$ & \\
\hline $\mathrm{G} 2$ & 96 & $61(64)$ & $35(36)$ & \\
\hline G3 & 41 & $20(49)$ & $21(51)$ & \\
\hline pT status & & & & 0.025 \\
\hline pT1 & 43 & $34(79)$ & $9(21)$ & \\
\hline pT2 & 34 & $21(62)$ & $13(38)$ & \\
\hline pT3 & 88 & $48(55)$ & $40(45)$ & \\
\hline pN status & & & & $<0.001$ \\
\hline pNO & 81 & $62(77)$ & $19(23)$ & \\
\hline $\mathrm{pN} 1$ & 84 & $41(49)$ & $43(51)$ & \\
\hline pM status & & & & 0.003 \\
\hline $\mathrm{pMX}$ & 140 & $94(67)$ & $46(33)$ & \\
\hline pM1 & 25 & $9(36)$ & $16(64)$ & \\
\hline FIGO stage & & & & $<0.001$ \\
\hline I & 29 & $25(86)$ & $4(14)$ & \\
\hline II & 20 & $16(80)$ & $4(20)$ & \\
\hline III & 91 & $53(58)$ & $38(42)$ & \\
\hline IV & 25 & $9(36)$ & $16(64)$ & \\
\hline
\end{tabular}

${ }^{\mathrm{a}} \chi^{2}$ test; ${ }^{\mathrm{b}}$ Mean age; ${ }^{\mathrm{C} E n d o m e t r i o i d, ~ c l e a r ~ c e l l ~ a n d ~ u n d i f f e r e n t i a t e d ~ t y p e s . ~ A G B L 2, ~ A T P / G T P ~ b i n d i n g ~ p r o t e i n ~ l i k e ~ 2 ; ~ F I G O, ~ I n t e r n a t i o n a l ~}$ Federation of Gynecology and Obstetrics.

Table II. AGBL2 expression in normal ovarian tissues and in benign and malignant epithelial ovarian tumor tissues.

\section{AGBL2 protein}

Tissue type

Number of cases, $n$

Low expression, $\mathrm{n}(\%)$

High expression, n $(\%)$

\begin{tabular}{lrrr}
\hline Normal ovaries & 30 & $30(100)$ & $0(0)$ \\
Cystadenomas & 35 & $32(91)$ & $3(9)$ \\
Borderline tumors & 38 & $30(79)$ & $8(21)$ \\
Invasive carcinomas & 165 & $103(62)$ & $62(38)$ \\
\hline
\end{tabular}

A significant increasing frequency of high expression of AGBL2 was observed in cystadenomas, in borderline tumors and in invasive carcinomas $\left(\mathrm{P}<0.01, \chi^{2}\right.$-test for trend).

pathological tumor-node-metastasis ( $\mathrm{pT} / \mathrm{pN} / \mathrm{pM})$ status and FIGO stage in patients with ovarian carcinoma $(\mathrm{P}<0.05$; Table I). No significant association was observed between
AGBL2 expression and other clinicopathological characteristics, including the patient age and tumor histological type (P>0.05; Table I). 
Table III. Analysis of the clinicopathological characteristics and AGBL2 expression for the prognosis of 165 patients with ovarian carcinoma by univariate survival analysis (log-rank test).

\begin{tabular}{|c|c|c|c|c|}
\hline Variable & Number of cases, $\mathrm{n}$ & Mean survival, months & Median survival, months & P-value \\
\hline Age at surgery, years & & & & 0.949 \\
\hline$\leq 51.0^{\mathrm{a}}$ & 88 & 76.6 & 64.0 & \\
\hline$>51.0^{\mathrm{a}}$ & 77 & 63.9 & 62.0 & \\
\hline Histological type & & & & 0.528 \\
\hline Serous & 109 & 64.6 & 52.0 & \\
\hline Mucinous & 19 & 72.5 & 70.0 & \\
\hline Others $^{\mathrm{c}}$ & 37 & 97.6 & $\mathrm{NR}^{\mathrm{b}}$ & \\
\hline Histological grade (Silveberg) & & & & 0.001 \\
\hline G1 & 28 & 110.1 & NR & \\
\hline G2 & 96 & 73.3 & 64.0 & \\
\hline G3 & 41 & 43.6 & 28.0 & \\
\hline pT status & & & & 0.001 \\
\hline pT1 & 43 & 111.8 & NR & \\
\hline pT2 & 34 & 76.8 & 66.0 & \\
\hline pT3 & 88 & 55.9 & 34.0 & \\
\hline pN status & & & & $<0.001$ \\
\hline $\mathrm{pN} 0$ & 81 & 89.1 & 128.0 & \\
\hline $\mathrm{pN} 1$ & 84 & 52.5 & 30.0 & \\
\hline pM status & & & & $<0.001$ \\
\hline $\mathrm{pMX}$ & 140 & 85.3 & 128.0 & \\
\hline pM1 & 25 & 22.1 & 13.0 & \\
\hline FIGO stage & & & & $<0.001$ \\
\hline I & 29 & 134.2 & NR & \\
\hline II & 20 & 107.6 & NR & \\
\hline III & 91 & 60.3 & 36.0 & \\
\hline IV & 25 & 22.1 & 13.0 & \\
\hline AGBL2 expression & & & & $<0.001$ \\
\hline Low expression & 103 & 91.2 & 128.0 & \\
\hline High expression & 62 & 46.4 & 22.0 & \\
\hline
\end{tabular}

${ }^{\mathrm{a}}$ Mean age; ${ }^{\mathrm{b}}$ Not reached; ${ }^{\mathrm{c}}$ Endometrioid, cear cell and undifferentiated types. AGBL2, ATP/GTP binding protein like 2; FIGO, International Federation of Gynecology and Obstetrics.

The cumulative survival curves using Kaplan-Meier method for univariate survival analysis were calculated, and the differences in survival times were analyzed by the log-rank test. The results demonstrated that the prognostic predictors of patient survival, including tumor histological grade $(\mathrm{P}=0.001), \mathrm{pT} / \mathrm{pN} / \mathrm{pM}$ status $(\mathrm{P}<0.01)$ and FIGO stage $(\mathrm{P}<0.001)$, had a significant impact on patient prognosis (Table III), which confirmed that the cohort of patients presented in the present study was representative of the general population with ovarian carcinoma. Subsequently, the impact of AGBL2 expression level on the survival of patients with ovarian carcinoma was analyzed. The results revealed that the mean survival time of patients with ovarian carcinoma and high AGBL2 expression was of 46.4 months, which was significantly shorter than the 91.2 months for patients with low AGBL2 expression ( $\mathrm{P}<0.001$; Table III; Fig. 2).

In order to determine whether the variables exhibiting prognostic influence following univariate analysis were covariates, the expression of AGBL2 and other significant clinicopathological characteristics, including tumor histological grade, $\mathrm{pT} / \mathrm{pN} / \mathrm{pM}$ status, and FIGO stage, were assessed with multivariate analysis (Table IV). Multivariate Cox regression analysis revealed that AGBL2 expression $(\mathrm{P}=0.004), \mathrm{pN}$ status $(\mathrm{P}=0.034)$ and FIGO stage $(\mathrm{P}<0.001)$ were independent prognostic factors for poor overall survival (Table IV).

ROC can maximize both sensitivity and specificity for the outcome and can therefore highlight the point on the curve closest to $(0.0,1.0)$ for each clinicopathologic characteristic. In order to evaluate the patient survival status, ROC curve analysis was performed for each clinicopathologic characteristic and AGBL2 expression (area under the curve $=0.645 ; \mathrm{P}=0.001$; Fig. 3 ).

Association between AGBL2, IRGM and LC $3 A / B$ expression in patients with ovarian carcinoma. A recent study reported that AGBL2 participates in tumorigenesis 
Table IV. Multivariate analysis of the overall survival of patients with ovarian carcinoma (Cox regression model).

\begin{tabular}{lccc}
\hline Variable & Relative risk & $\begin{array}{c}95 \% \text { confidence } \\
\text { interval }\end{array}$ & P-value \\
\hline AGBL2 $^{\mathrm{a}}$ & 2.052 & $1.262-3.337$ & 0.004 \\
pN status $^{\mathrm{b}}$ & 1.727 & $1.042-2.861$ & 0.034 \\
FIGO stage $^{\mathrm{c}}$ & 3.169 & $2.161-4.646$ & 0.000 \\
\hline
\end{tabular}

${ }^{\mathrm{a}}$ High expressin vs. low expression; ${ }^{\mathrm{b}} \mathrm{pN} 1$ vs. pN0; 'Stage IV vs. stage III vs. stage II vs. stage I. AGBL2, ATP/GTP binding protein like 2; FIGO, International Federation of Gynecology and Obstetrics.
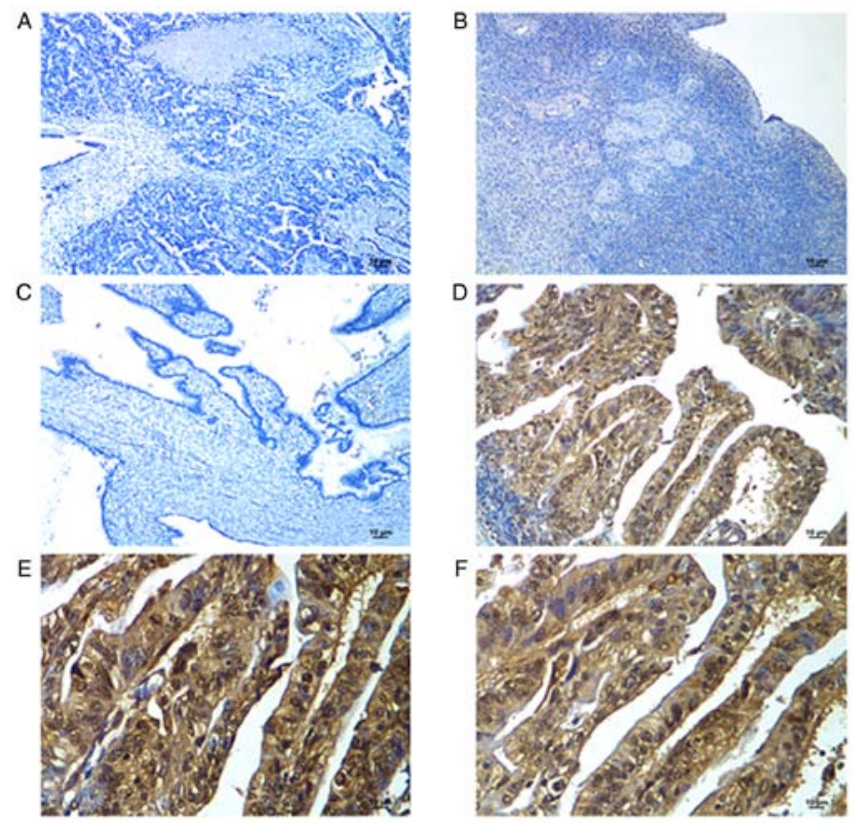

Figure 1. Immunohistochemical staining of AGBL2 in human ovarian tissues and of IRGM and LC3A/B in epithelial ovarian cancer tissue. (A) Negative control was obtained by incubating tissue with normal IgG. Magnification, x100. Scale bar, $10 \mu \mathrm{m}$. (B) Negative expression of AGBL2 was observed in the surface epithelium of normal ovarian tissue Magnification, x100. Scale bar, $10 \mu \mathrm{m}$. (C) Ovarian cystadenoma presented low AGBL2 expression (10\% of tumor cells showed moderate positive staining). Magnification, x100. Scale bar, $10 \mu \mathrm{m}$. (D) High AGBL2 expression was detected in ovarian carcinoma tissue (case 52; $>90 \%$ of carcinoma cells showed strong positive). Magnification, x200. Scale bar, $10 \mu \mathrm{m}$. High expression of (E) IRGM and (F) LC3A/B in ovarian carcinoma (case 52; $>90 \%$ of carcinoma cells showed strong positive staining for these two proteins). Magnification, $\mathrm{x} 400$. Scale bar, $10 \mu \mathrm{m}$. AGBL2, ATP/GTP binding protein like 2; IRGM, immunity related GTPase M.

through IRGM-regulated autophagy (18). The potential association between AGBL2 expression and IRGM and LC3A/B expression was therefore assessed by IHC in tissues from patients with ovarian carcinoma (Fig. 1D-F). The results demonstrated that AGBL2 expression level was significantly associated with IRGM $(\mathrm{P}=0.013)$ and LC3A/B $(\mathrm{P}=0.004)$ expression level. In addition, IRGM expression level was positively associated with LC3A/B expression level $(\mathrm{P}=0.023$; Table V).

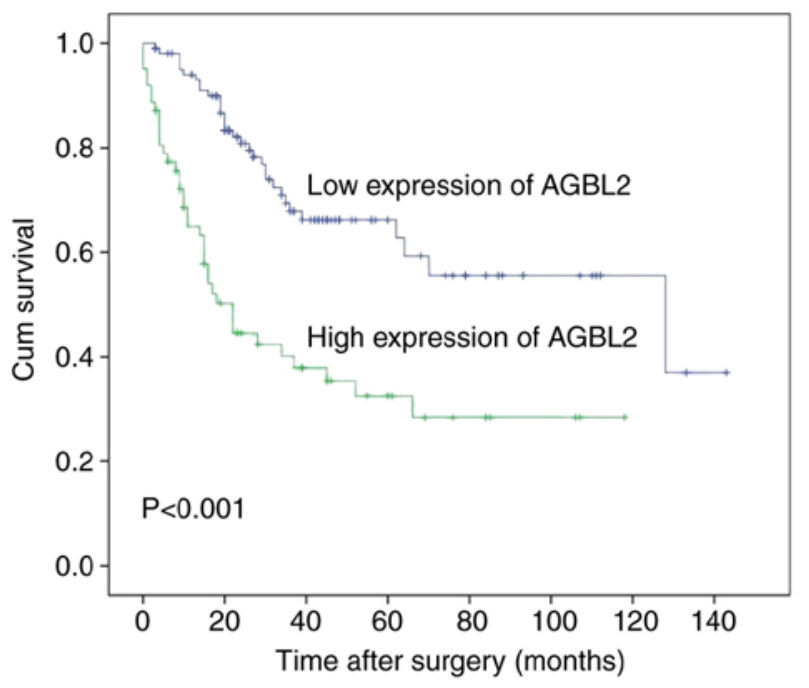

Figure 2. Kaplan-Meier survival analysis of AGBL2 expression in 165 patients with invasive ovarian carcinoma (log-rank test). The overall survival and probability of survival of patients with ovarian carcinoma were investigated. High expression of AGBL2, $n=62$; low expression of AGBL2, $\mathrm{n}=103(\mathrm{P}<0.001)$. AGBL2, ATP/GTP binding protein like 2 .

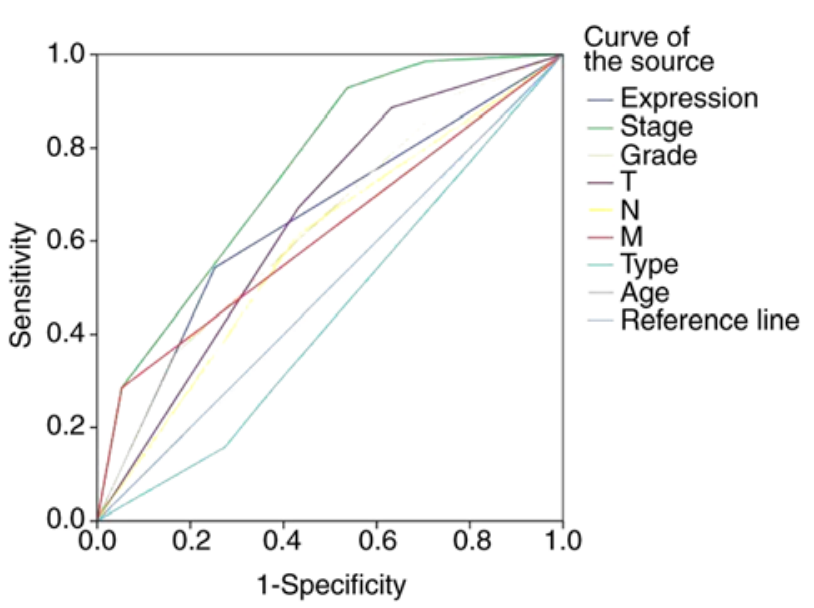

Figure 3. Receiver operating characteristic curve analysis on different clinicpathological characteristics and AGBL2 expression was performed to evaluate survival status of patients with ovarian carcinoma. AGBL2 protein expression $(\mathrm{AUC}=0.645 ; \mathrm{P}=0.001)$, International Federation of Gynecology and Obstetrics stage $(\mathrm{AUC}=0.755 ; \mathrm{P}<0.001)$, pT $(\mathrm{AUC}=0.648 ; \mathrm{P}=0.001)$, $\mathrm{pN}(\mathrm{AUC}=0.591 ; \mathrm{P}=0.045), \mathrm{pM}(\mathrm{AUC}=0.617 ; \mathrm{P}=0.011)$, pathological grading ( $\mathrm{AUC}=0.633 ; \mathrm{P}=0.004)$ were significantly associated with patients survival. AGBL2, ATP/GTP binding protein like 2; AUC, area under the curve.

\section{Discussion}

Epithelial ovarian cancer is the most lethal gynecological malignancy in postmenopausal women (4). Although remarkable efforts have been made to improve treatment modalities, ovarian carcinoma remains the primary cause of gynecological cancer-associated mortality (5). It is therefore crucial to identify novel therapeutic approaches and targets to improve early diagnosis and patient outcome.

Recent reports have verified that AGBL2 catalyzes MT detyrosination and promotes tumorigenesis and cancer progression (8). However, to date, the expression status of AGBL2 and its clinicopathological significance in ovarian 
Table V. Association between AGBL2 expression and IRGM and LC3A/B expression in ovarian carcinoma tissues.

\begin{tabular}{|c|c|c|c|c|c|c|c|}
\hline \multirow[b]{2}{*}{ Expression level } & \multirow[b]{2}{*}{ Number of cases, $n$} & \multicolumn{3}{|c|}{ IRGM expression } & \multicolumn{3}{|c|}{ LC3A/B expression } \\
\hline & & Low, n (\%) & High, n (\%) & P-value & Low, n (\%) & High, n (\%) & P-value ${ }^{a}$ \\
\hline AGBL2 expression & & & & 0.013 & & & 0.004 \\
\hline Low & 103 & $35(34)$ & $68(66)$ & & $65(63)$ & $38(37)$ & \\
\hline High & 62 & $10(16)$ & $52(84)$ & & $25(40)$ & $37(60)$ & \\
\hline LC3A/B expression & & & & 0.023 & & & \\
\hline Low & 90 & $31(34)$ & $59(66)$ & & & & \\
\hline High & 75 & $14(19)$ & $61(81)$ & & & & \\
\hline
\end{tabular}

${ }^{a} \chi^{2}$ test. ATP/GTP binding protein like 2; IRGM, immunity related GTPase M.

carcinomas has not been elucidated (12-14). In the present study, IHC was used to investigate whether an abnormal AGBL2 expression could be involved in the pathogenesis of ovarian carcinoma. By using a large cohort of ovarian tissues, including normal, benign and borderline epithelial ovarian tumor tissues, and malignant epithelial cancers, the results demonstrated that AGBL2 high expression level was consistent with ovarian tumor malignancy. AGBL2 was more frequently highly expressed in ovarian carcinomas, compared within benign and borderline tumors. Furthermore, high AGBL2 expression was associated with high histological grade and advanced clinical stage (pT/pN/pM and FIGO stage). These results confirmed that AGBL2 could serve a crucial role in tumorigenesis promotion of ovarian carcinoma. Previous studies demonstrated that AGBL2 high expression is a prognostic marker of cancer and is associated with poor clinical outcome in patients with hepatocellular carcinoma and breast cancer $(18,19)$. Similarly, the results from this study indicated that AGBL2 high expression could predict poor overall survival in patients with ovarian carcinomas, and that AGBL2 may be considered as an independent prognostic marker. Altogether, these findings suggest that AGBL2 high expression may serve as a reliable prognosis biomarker in patients with ovarian carcinoma. Subsequently, IHC staining of AGBL2 may be used as an additional tool to identify whether patients with ovarian carcinoma could have an increased risk of tumor metastasis and/or a poor prognosis.

A recent study reported that AGBL2 stimulates autophagy by regulating IRGM and therefore promote cancer cell proliferation (18). The potential association between AGBL2 expression and IRGM and LC3A/B expression was therefore evaluated by IHC in tissues from patients with ovarian carcinoma. The results demonstrated that AGBL2 expression level was significantly associated IRGM and LC3A/B expression level in ovarian carcinoma tissues. Furthermore, IRGM expression level was positively associated with LC3A/B expression level. These findings indicated that AGBL2 might also regulate autophagy by modulating IRGM in ovarian carcinoma. A previous study reported that inhibition of $\mathrm{PI} 3 \mathrm{~K} / \mathrm{AKT} / \mathrm{mTOR}$ and the Ras/MAPK signaling pathways, which are involved in autophagy activation, can limit the development of ovarian cancer (23). The results from the present study identified a potential molecular mechanism for the oncogenic role of AGBL2 in ovarian carcinoma pathogenesis, and suggested that AGBL2 may enhance autophagy to support the malignant phenotype of cancer cells. However, this study was a retrospective study, and fresh tissue samples were only rarely collected. The limitation of this study was the exclusive use of IHC to determine AGBL2, IRGM and LC3A/B expression levels. Future studies incorporating western blotting, reverse-transcription-quantitative PCR and cell line experiments are required for further investigation.

In conclusion, the present study was the first to investigated AGBL2 expression in a large cohort of normal human ovarian tissue, and in benign, borderline and malignant epithelial ovarian tumors tissues by IHC. The results demonstrated that AGBL2 expression was upregulated in tumor tissues and may therefore serve as a novel, reliable and independent prognostic marker in ovarian carcinoma. In addition, these results further confirmed that increased AGBL2 expression may enhance the aggressive behavior of ovarian carcinoma.

\section{Acknowledgements}

Not applicable.

\section{Funding}

The present study was funded by the Nature Science Foundation of China (grant no. 81772769) and the Research Project in the Science and Technology Bureau in Guangzhou (grant no. 201704020125).

\section{Availability of data and materials}

The datasets used and/or analyzed during the current study are available from the corresponding author on reasonable request.

\section{Authors' contributions}

WPH and LLW designed and performed the experiments and drafted and revised the manuscript. Both authors approved the final version of the manuscript. 


\section{Ethics approval and consent to participate}

The present study was approved by the Research Ethics Committee of the First Affiliated Hospital, Sun Yat-Sen University (approval no. 2018-32) and all patients provided written informed consent prior to the study.

\section{Patient consent for publication}

Not applicable.

\section{Competing interests}

The authors declare that they have no competing interests.

\section{References}

1. Wingo PA, Ries LA, Rosenberg HM, Miller DS and Edwards BK Cancer incidence and mortality. 1973-1995, a report card for the U.S. Cancer 82: 1197-1207, 1998.

2. Siegel RL, Miller KD and Jemal A: Cancer statistics, 2018. CA Cancer J Clin 68: 7-30, 2018.

3. Coburn SB, Bray F, Sherman ME and Trabert B: International patterns and trends in ovarian cancer incidence, overall and by histologic subtype. Int J Cancer 140: 2451-2460, 2017.

4. Jelovac D and Armstrong DK: Recent progress in the diagnosis and treatment of ovarian cancer. CA Cancer J Clin 61: 183-203, 2011.

5. Oza AM, Cook AD, Pfisterer J, Embleton A, Ledermann JA, Pujade-Lauraine E, Kristensen G, Carey MS, Beale P, Cervantes A, et al: Standard chemotherapy with or without bevacizumab for women with newly diagnosed ovarian cancer (ICON7): Overall survival results of a phase 3 randomised trial. Lancet Oncol 16: 928-936, 2015.

6. Coleman RL, Monk BJ, Sood AK and Herzog TJ: Latest research and treatment of advanced-stage epithelial ovarian cancer. Nat Rev Clin Oncol 10: 211-224, 2013.

7. Barnholtz-Sloan JS, Schwartz AG, Qureshi F, Jacques S, Malone J and Munkarah AR: Ovarian cancer: Changes in patterns at diagnosis and relative survival over the last three decades. Am J Obstet Gynecol 189: 1120-1127, 2003.

8. Sahab ZJ, Hall MD, Me Sung Y, Dakshanamurthy S, Ji Y, Kumar D and Byers SW: Tumor suppressor RARRES1 interacts with cytoplasmic carboxypeptidase AGBL2 to regulate the $\alpha$-tubulin tyrosination cycle. Cancer Res 71: 1219-1228, 2011.

9. de Forges H, Bouissou A and Perez F: Interplay between microtubule dynamics and intracellular organization. Int $\mathrm{J}$ Biochem Cell Biol 44: 266-274, 2012.

10. Song Y and Brady ST: Post-translational modifications of tubulin: Pathways to functional diversity of microtubules. Trends Cell Biol 25: 125-136, 2015.

11. Webster DR, Gundersen GG, Bulinski JC and Borisy GG: Differential turnover of tyrosinated and detyrosinated microtubules. Proc Natl Acad Sci USA 84: 9040-9044, 1987.
12. Kato C, Miyazaki K, Nakagawa A, Ohira M, Nakamura Y, Ozaki T, Imai T and Nakagawara A: Low expression of human tubulin tyrosine ligase and suppressed tubulin tyrosination/detyrosination cycle are associated with impaired neuronal differentiation in neuroblastomas with poor prognosis. Int J Cancer 112: 365-375, 2004.

13. Mialhe A, Lafanechère L, Treilleux I, Peloux N, Dumontet $C$, Brémond A, Panh MH, Payan R, Wehland J, Margolis RL and Job D: Tubulin detyrosination is a frequent occurrence in breast cancers of poor prognosis. Cancer Res 61: 5024-5027, 2001.

14. Soucek K, Kamaid A, Phung AD, Kubala L, Bulinski JC, Harper RW and Eiserich JP: Normal and prostate cancer cells display distinct molecular profiles of alpha-tubulin posttranslational modifications. Prostate 66: 954-965, 2006.

15. Whipple RA, Matrone MA, Cho EH, Balzer EM, Vitolo MI, Yoon JR, Ioffe OB, Tuttle KC, Yang J and Martin SS: Epithelial-to-mesenchymal transition promotes tubulin detyrosination and microtentacles that enhance endothelial engagement. Cancer Res 70: 8127-8137, 2010.

16. Whipple RA, Balzer EM, Cho EH, Matrone MA, Yoon JR and Martin SS: Vimentin filaments support extension of tubulin-based microtentacles in detached breast tumor cells. Cancer Res 68: 5678-5688, 2008

17. Gundersen GG and Bulinski JC: Selective stabilization of microtubules oriented toward the direction of cell migration. Proc Natl Acad Sci USA 85: 5946-5950, 1988.

18. Wang LL, Jin XH, Cai MY, Li HG, Chen JW, Wang FW, Wang $\mathrm{CY}, \mathrm{Hu} \mathrm{WW}$, Liu $\mathrm{F}$ and Xie D: AGBL2 promotes cancer cell growth through IRGM-regulated autophagy and enhanced Aurora A activity in hepatocellular carcinoma. Cancer Lett 414: 71-80, 2018

19. Zhang H, Ren Y, Pang D and Liu C: Clinical implications of AGBL2 expression and its inhibitor latexin in breast cancer. World J Surg Oncol 12: 142, 2014.

20. Yang GF, Li XM and Xie D: Overexpression of clusterin in ovarian cancer is correlated with impaired survival. Int J Gynecol Cancer 19: 1342-1346, 2009.

21. He WP, Zhou J, Cai MY, Xiao XS, Liao YJ, Kung HF, Guan XY, Xie D and Yang GF: CHD1L protein is overexpressed in human ovarian carcinomas and is a novel predictive biomarker for patients survival. BMC Cancer 12: 437, 2012

22. Xu L, Li X, Cai M, Chen J, Li X, Wu WK, Kang W, Tong J, To KF, Guan XY, et al: Increased expression of Solute carrier family 12 member 5 via gene amplification contributes to tumour progression and metastasis and associates with poor survival in colorectal cancer. Gut 65: 635-646, 2016.

23. Lu Z, Yang H, Sutton MN, Yang M, Clarke CH, Liao WS and Bast RC Jr: ARHI (DIRAS3) induces autophagy in ovarian cancer cells by downregulating the epidermal growth factor receptor, inhibiting PI3K and Ras/MAP signaling and activating the FOXo3a-mediated induction of Rab7. Cell Death Differ 21: $1275-1289,2014$

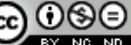

This work is licensed under a Creative Commons Attribution-NonCommercial-NoDerivatives 4.0 International (CC BY-NC-ND 4.0) License. 